\title{
Gender-Related Survival in Different Stages of Lung Cancer-A Population Study over 20 Years
}

\author{
Gunnar Svensson 1,2*, Sven-Börje Ewers ${ }^{3}$, Ola Ohlsson', Håkan Olsson ${ }^{2,3}$ \\ ${ }^{1}$ Department of Medicine, Central Hospital, Kristianstad, Sweden \\ ${ }^{2}$ Department of Cancer Epidemiology, Clinical Sciences, University of Lund, Lund, Sweden \\ ${ }^{3}$ Department of Oncology, University Hospital, Lund, Sweden \\ Email: "gunnar.a.svensson@telia.com
}

Received 17 June 2014; revised 16 July 2014; accepted 16 August 2014

Copyright (C) 2014 by authors and Scientific Research Publishing Inc.

This work is licensed under the Creative Commons Attribution International License (CC BY).

http://creativecommons.org/licenses/by/4.0/

(c) (i) Open Access

\section{Abstract}

Introduction: Tumour stage is the most important prognostic factor in non-small cell lung cancer (NSCLC) and small cell lung cancer (SCLC). The aim of this study was to evaluate if female gender was a prognostic factor in different tumour stages in relation to histology and given therapy. Methods: From 1989-2008, 1497 patients in eastern Scania, in southern Sweden with 202,000 inhabitants, were referred and prospectively registered. Tumour stage, performance status, lung cancer type and primary therapy were registered. Results: In NSCLC, female patients in stages 1 and 2 who were treated with surgery had a better 5-year survival rate $(\mathbf{7 9 . 4 \% )}$, compared to males $(60.6 \% ; p=0.0004)$. Female patients in stage 3 with active therapy (surgery and/or radiotherapy and/or chemotherapy) had a better 5 -year survival than males $(20.6 \%$ vs. $10.5 \%$, respectively, $p=0.0006$ ). Female patients with adenocarcinoma were favourable in stages 1-3. In stage 4, there was no survival difference between females and males. In SCLC, females with limited disease (LD) and active therapy (chemotherapy \pm radiotherapy \pm surgery) had a higher 5-year survival rate $(28 \%)$ than males $(5.6 \%) ; p=0.001$. Females with extensive disease (ED) and active therapy (chemotherapy \pm radiotherapy) had a better 5 -year survival $(3.9 \%)$ compared to males $(0.7 \%)$; $p$ $=0.023$. In multivariate analyses, patient performance status at diagnosis was also an independent prognostic factor in all tumour stages of lung cancer. Conclusions: This population-based study corroborates a female survival advantage in NSCLC stages 1-3, but not in metastatic stage 4 , and this is also demonstrated for the adenocarcinoma subgroup. The study also confirms better prognosis in females with SCLC in both LD and ED. The study also demonstrates the importance of patient performance status as a prognostic factor in all stages of lung cancer.

${ }^{*}$ Corresponding author.

How to cite this paper: Svensson, G., Ewers, S.-B., Ohlsson, O. and Olsson, H. (2014) Gender-Related Survival in Different Stages of Lung Cancer-A Population Study over 20 Years. Open Journal of Internal Medicine, 4, 47-58.

http://dx.doi.org/10.4236/ojim.2014.43008 


\section{Keywords}

\section{Gender, Performance Status, Prognostic Factor, NSCLC (Non-Small Cell Lung Cancer), SCLC (Small Cell Lung Cancer), Tumour Stage}

\section{Background}

Lung cancer patients have a low 5-year survival rate of about 10\% - 15\% in many Western countries. The most important clinical factors for survival are low tumour stage and surgical resection [1].

Good patient performance status has also emerged as an important clinical factor [2].

Other factors, such as the histological SCLC subtype, are linked to a worse prognosis with early dissemination, but on the other hand, are associated with greater chemo-sensitivity than NSCLC [3].

Older age and former and current smoking history have also been associated with worse prognosis [4].

Patient gender, with a better prognosis seen in females, has been recognized as a significant factor in SCLC [5] since the 1980s, and since the 1990s in NSCLC patients [6].

In SCLC, female patients with limited disease (LD) have generally had a better prognosis than males [7].

In NSCLC, females have generally had better survival, especially in patients in early tumour stages and those who have undergone surgical resection [6].

In our recent population based study [8], the prognosis in female patients with lung cancer was favourable compared to males both in NSCLC and SCLC.

The aim of the present study was to analyse gender differences in survival in different tumour stages with regard to lung cancer types and primary treatment combinations in a prospectively sampled population study over two decades (1989-2008).

\section{Materials and Methods}

The materials and methods used here have been described in detail in a study by Svensson et al. [8]. The eastern part of Scania in southern Sweden contains one Central Hospital and two local hospitals for a population of approximately 202,000 residents. The total population numbers for the time period included in this study were obtained from Statistics Sweden [9].

The coverage of the total referral area was $85.9 \%$ (1497/1743), while for the major part of the area, 91.7\% (1370/1494) was covered. In a smaller part of the area (consisting of the Simrishamn and Tomelilla communities), the coverage was only $51 \%$ (127/249) because some patients were sent directly to the Lund University Hospital and other patients were treated at the local hospital.

This research project was approved by the Lund University Ethic Committee (reg.nr. 2009/225), Sweden.

\subsection{The Local Registry and Patient Characteristics}

A local registry of lung cancer patients was consecutively established for all patients diagnosed at or referred to the Pulmonary Section, Department of Medicine, Central Hospital, Kristianstad between the years 1989-2008. One of the authors (GS) was responsible for patient registration and was involved in the diagnosis and/or treatment of most of the patients, thus ensuring the consistency of the data collection. The local registry was a clinical based quality registry and patients were informed and could refrain to participate. All patients were Caucasians.

The local registry lists the type of lung cancer, e.g., squamous cell carcinoma, small cell carcinoma, adenocarcinoma, large cell carcinoma, adenosquamous cancer, carcinoid tumour, adenoid cystic carcinoma, other lung cancer and clinical lung cancer, according to the World Health Organization classification (WHO 1981) [10]. The date of diagnosis was set as the date when data from the pathological classification were available.

The tumour-node-metastases classification and staging (1-4) for NSCLC were established according to the international staging system for lung cancer (1986) [11]. A revision of the staging system was introduced in 1997 [1], when T3N0 tumours were reclassified from 3A to 2B. For primary surgery patients with NSCLC, the pathological stage was used, while in others, the clinical stage was applied. In SCLC, the Veterans Administra- 
tion staging system [12] of limited disease (LD) and extensive disease (ED) was used.

The patient performance status at diagnosis was recorded according to the Eastern Cooperative Oncology Groups (ECOG) 0-4 [13]. The performance status group was recorded in the registration form, and the description of patient activity and symptoms were recorded in the hospital medical record.

The data of patients not referred to Central Hospital with lung cancer in our study were obtained from the Regional Tumour Registry in Lund. Only the lung cancer types were available. The time of death for patients not in the registry was acquired from the population registry.

\subsection{Statistical Analyses}

Analyses are based on 1497 patients in the local registry.

The chi-squared test was used for statistical comparisons of different factors (age, lung cancer types) between females and males in the respective tumour stages. The Mann-Whitney two-sided test was used to compare performance status groups between genders in the respective tumour stages. Survival was analysed with KaplanMeier survival curves and life-tables, and differences were assessed by the two-sided log-rank test. For every tumour stage, univariate and multivariate prognostic factors (age, gender, performance status, time period, primary therapy combination and, in NSCLC, lung cancer type) were analysed with Cox proportional hazard regression. A further stratification for ages $<70$ and ages $\geq 70$ (in every tumour stage), and with age as a continuous variable, were analyzed to control for more comorbidities in ages $\geq 70$ and a longer natural survival in females. All patients were followed until 2009-07-01 or until death. Statistica version 10.0 (StatSoft, Tulsa, OK, USA) was used for all statistical analyses.

\section{Results}

Patients in the local registry $(\mathrm{n}=1497)$, distribution of stages and lung cancer types are outlined in Figure 1. NSCLC was seen in $82 \%$ and SCLC in $18 \%$. In NSCLC, adenocarcinoma was most frequent $49 \%$ and stage 4 in $42 \%$. Because of the small number of patients in stage $2(6.7 \%)$ they were pooled together with stage 1.

\subsection{Gender and Clinical Factors}

Female patients increased their relative frequency from $26 \%$ from the first period $1989-2003$ to $48 \%$ in the last period 2003-2008 $(\mathrm{p}=0.017)$.

There were more female patients younger than age 70 in all lung cancer stages, but this age difference was significant $(\mathrm{p}=0.016)$ only in NSCLC stage $1-2$ (Table 1$)$.

No significant gender difference in performance status distribution was seen in any stage.

Squamous cell carcinoma was significantly $(\mathrm{p}<0.001)$ more frequent in males in all stages. In females, adenocarcinoma was more frequent in all tumour stages $(\mathrm{p}<0.001)$.

Female patients had more surgery in NSCLC stages 1-3, but this difference was not significant.

\subsection{Survival in NSCLC}

Female patients in stage 1-2, who were treated with surgery $(\mathrm{n}=99)$, had a better 5 -year survival compared to males $(n=119)(79.4 \%$ vs. $60.6 \%$, respectively; $\mathrm{p}=0.0004)$. In patients ( 14 females, 24 males) treated with radiotherapy \pm chemotherapy there were no survival differences between males and females. Survival curves in actively treated patients, see Figure 2. In the subgroup of actively treated patients with adenocarcinoma the 5 -year survival rate was $79 \%$ compared to $60 \%$ in males $(\mathrm{p}=0.012)$. In patients with squamous cell carcinoma the 5 -year survival rate was $46 \%$ in females and $52 \%$ in males, n.s.

Female stage 3 patients who were treated with active therapy (surgery and/or radiotherapy and/or chemotherapy $(n=110)$ had a better 5 -year survival compared to males $(n=193)(20.6 \%$ vs. $10.5 \%$, respectively; $\mathrm{p}=$ 0.006). This female survival advantage was also significant in both adenocarcinoma and squamous cell carcinoma subgroups.

In stage 4 there was no survival difference between female and male patients who underwent active therapy (148 females, 177 males), and this was also the result in the adenocarcinoma and squamous cell carcinoma subgroups. There was no survival difference in patients who received no therapy (74 females, 121 males). 


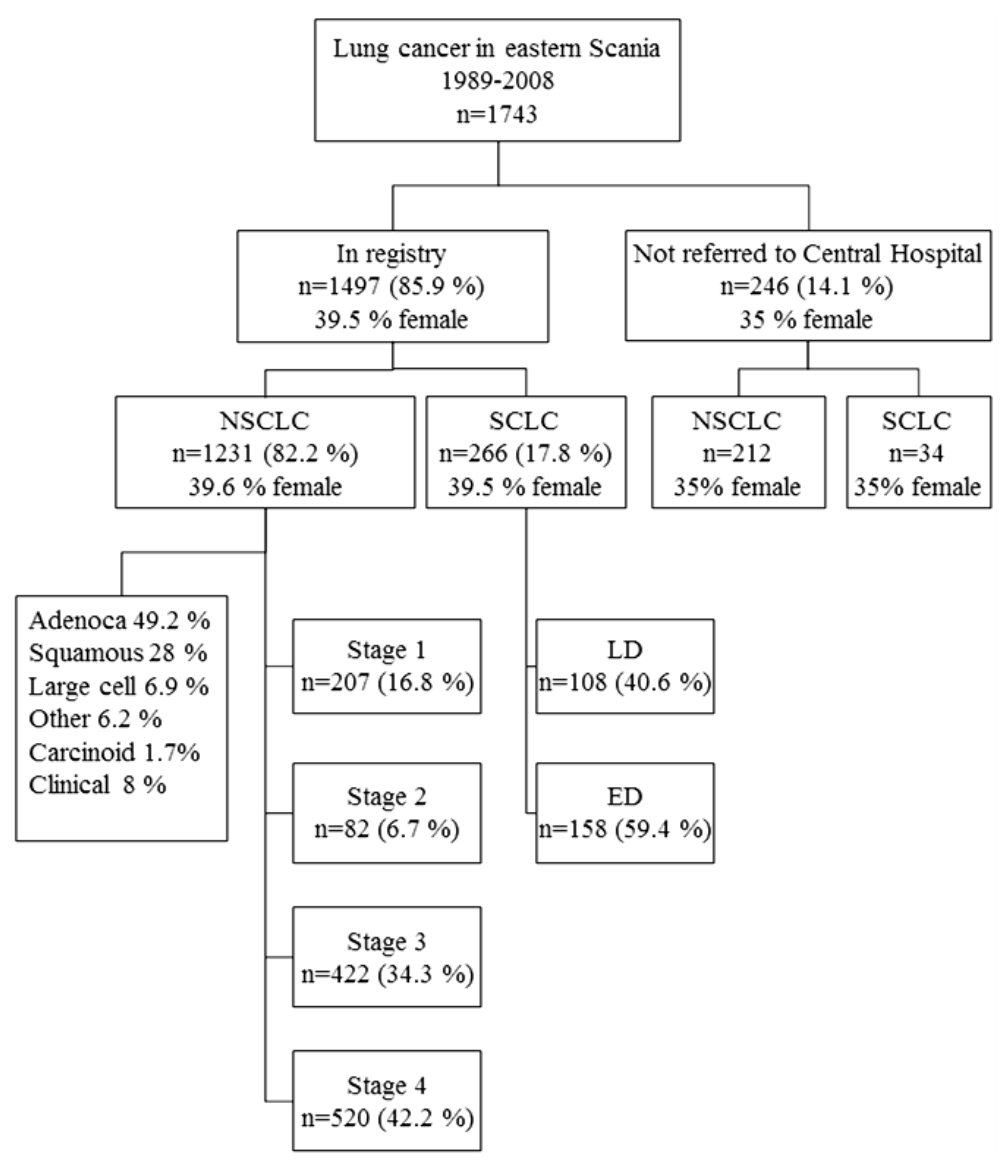

Figure 1. Patients diagnosed with lung cancer in eastern Scania from 19892008 by number (\%), gender, tumour type and stage [non-small cell lung carcinoma (NSCLC) 1-4, small cell lung carcinoma (SCLC) limited disease (LD)/ extensive disease (ED)]. Adenoca, adenocarcinoma; Squamous, squamous cell carcinoma; Large, large cell carcinoma; Other, other lung cancer; Clinical, clinical lung cancer. With permission from John Wiley \& Sons, Inc. [8].

\subsection{Survival in SCLC}

Patients with LD who were treated with surgery + chemotherapy had a better 5-year survival (35.6\%) compared to patients treated with chemoradiation $(15.6 \%)(p=0.025)$.

As shown in Figure 3, female patients with LD and active therapy (chemotherapy \pm radiotherapy \pm surgery) had a better 5-year survival $(n=47 ; 28 \%)$ than males $(n=57 ; 5.6 \%)(p=0.001)$.

In patients with ED and active therapy (chemotherapy \pm or radiotherapy) females had a better 2-year ( $\mathrm{n}=50$; $13.8 \%)$ and 5-year (3.9\%) survival compared to males $(\mathrm{n}=78 ; 2.6 \%$ and $0.7 \%$, respectively) $(\mathrm{p}=0.023)$.

\subsection{Multivariate Analyses}

Male patients had a worse prognosis than females in all lung cancer stages, except NSCLC stage 4, when adjusted for age, performance status, lung cancer type (in NSCLC) and primary therapy combinations (Table 2 and Table 3). This result was also found in a stratified analysis of patients aged $<70$ years (with age as a continuous variable). In patients $\geq 70$ years of age, males with NSCLC stages 1-3 had a worse prognosis than females. Male patients $\geq 70$ years with SCLC and LD had a non-significant worse prognosis with a Hazard ratio (HR) 2.11 (95\% confidence interval (CI) $0.84-5.29$ ), $\mathrm{p}=0.110$, as had male patients $\geq 70$ years with ED, HR 1.73 (95\% CI 0.92 - 3.25), $\mathrm{p}=0.088$.

Patients with a performance status $>1$ vs. 0 - 1 had a worse prognosis in all lung cancer stages.

Patients at least 70 years old had a worse prognosis compared to individuals younger than 70 only in NSCLC 
Table 1. Demographics of lung cancer patients related to stage and gender in eastern Scania from 1989-2008.

\begin{tabular}{|c|c|c|c|c|c|c|c|c|c|c|}
\hline \multirow{3}{*}{$\begin{array}{c}\text { Stage: } \\
\text { Gender: }\end{array}$} & \multicolumn{6}{|c|}{ NSCLC } & \multicolumn{4}{|c|}{ SCLC } \\
\hline & \multicolumn{2}{|c|}{$1+2$} & \multicolumn{2}{|c|}{3} & \multicolumn{2}{|c|}{4} & \multicolumn{2}{|c|}{ LD } & \multicolumn{2}{|c|}{ ED } \\
\hline & Female & Male & Female & Male & Female & Male & Female & Male & Female & Male \\
\hline & n (\%) & n (\%) & n (\%) & n (\%) & n (\%) & n (\%) & n (\%) & n (\%) & n (\%) & n (\%) \\
\hline & $\begin{array}{c}124 \\
(43 \%)\end{array}$ & $\begin{array}{c}165 \\
(57 \%)\end{array}$ & $\begin{array}{c}141 \\
(33 \%)\end{array}$ & $\begin{array}{c}281 \\
(67 \%)\end{array}$ & $\begin{array}{c}222 \\
(43 \%)\end{array}$ & $\begin{array}{l}298 \\
(57 \%)\end{array}$ & $\begin{array}{c}49 \\
(45 \%)\end{array}$ & $\begin{array}{c}59 \\
(55 \%)\end{array}$ & $\begin{array}{c}56 \\
(35 \%)\end{array}$ & $\begin{array}{c}102 \\
(65 \%)\end{array}$ \\
\hline Age: $<70$ & $\begin{array}{c}68 \\
(55 \%)\end{array}$ & $\begin{array}{c}67 \\
(41 \%)\end{array}$ & $\begin{array}{c}68 \\
(48 \%)\end{array}$ & $\begin{array}{c}109 \\
(39 \%)\end{array}$ & $\begin{array}{c}130 \\
(59 \%)\end{array}$ & $\begin{array}{c}149 \\
(50 \%)\end{array}$ & $\begin{array}{c}35 \\
(71 \%)\end{array}$ & $\begin{array}{c}33 \\
(56 \%)\end{array}$ & $\begin{array}{c}34 \\
(61 \%)\end{array}$ & $\begin{array}{c}46 \\
(45 \%)\end{array}$ \\
\hline$\geq 70$ & $\begin{array}{c}56 \\
(45 \%)\end{array}$ & $\begin{array}{c}98 \\
(59 \%)\end{array}$ & $\begin{array}{c}73 \\
(52 \%)\end{array}$ & $\begin{array}{c}172 \\
(61 \%)\end{array}$ & $\begin{array}{c}92 \\
(41 \%)\end{array}$ & $\begin{array}{c}149 \\
(50 \%)\end{array}$ & $\begin{array}{c}14 \\
(29 \%)\end{array}$ & $\begin{array}{c}26 \\
(44 \%)\end{array}$ & $\begin{array}{c}22 \\
(39 \%)\end{array}$ & $\begin{array}{c}56 \\
(55 \%)\end{array}$ \\
\hline $\begin{array}{c}\text { Performance status: } \\
0-1\end{array}$ & $\begin{array}{l}105 \\
(85 \%)\end{array}$ & $\begin{array}{c}128 \\
(78 \%)\end{array}$ & $\begin{array}{c}86 \\
(61 \%)\end{array}$ & $\begin{array}{c}153 \\
(54 \%)\end{array}$ & $\begin{array}{c}71 \\
(32 \%)\end{array}$ & $\begin{array}{c}96 \\
(32 \%)\end{array}$ & $\begin{array}{c}30 \\
(61 \%)\end{array}$ & $\begin{array}{c}44 \\
(74 \%)\end{array}$ & $\begin{array}{c}17 \\
(30 \%)\end{array}$ & $\begin{array}{c}25 \\
(25 \%)\end{array}$ \\
\hline $2-4$ & $\begin{array}{c}19 \\
(15 \%)\end{array}$ & $\begin{array}{c}37 \\
(22 \%)\end{array}$ & $\begin{array}{c}55 \\
(39 \%)\end{array}$ & $\begin{array}{c}128 \\
(46 \%)\end{array}$ & $\begin{array}{c}151 \\
(68 \%)\end{array}$ & $\begin{array}{c}202 \\
(68 \%)\end{array}$ & $\begin{array}{c}19 \\
(39 \%)\end{array}$ & $\begin{array}{c}15 \\
(26 \%)\end{array}$ & $\begin{array}{c}39 \\
(70 \%)\end{array}$ & $\begin{array}{c}77 \\
(75 \%)\end{array}$ \\
\hline \multicolumn{11}{|l|}{ Time period: } \\
\hline $1989-1993$ & $\begin{array}{c}14 \\
(22 \%)\end{array}$ & $\begin{array}{c}51 \\
(78 \%)\end{array}$ & $\begin{array}{c}17 \\
(18 \%)\end{array}$ & $\begin{array}{c}80 \\
(82 \%)\end{array}$ & $\begin{array}{c}33 \\
(36 \%)\end{array}$ & $\begin{array}{c}59 \\
(64 \%)\end{array}$ & $\begin{array}{c}13 \\
(30 \%)\end{array}$ & $\begin{array}{c}31 \\
(70 \%)\end{array}$ & $\begin{array}{c}10 \\
(29 \%)\end{array}$ & $\begin{array}{c}24 \\
(71 \%)\end{array}$ \\
\hline 1994-1998 & $\begin{array}{c}30 \\
(42 \%)\end{array}$ & $\begin{array}{c}42 \\
(58 \%)\end{array}$ & $\begin{array}{c}24 \\
(28 \%)\end{array}$ & $\begin{array}{c}62 \\
(72 \%)\end{array}$ & $\begin{array}{c}38 \\
(38 \%)\end{array}$ & $\begin{array}{c}61 \\
(62 \%)\end{array}$ & $\begin{array}{c}13 \\
(54 \%)\end{array}$ & $\begin{array}{c}11 \\
(46 \%)\end{array}$ & $\begin{array}{c}12 \\
(31 \%)\end{array}$ & $\begin{array}{c}27 \\
(69 \%)\end{array}$ \\
\hline 1999-2003 & $\begin{array}{c}29 \\
(47 \%)\end{array}$ & $\begin{array}{c}33 \\
(53 \%)\end{array}$ & $\begin{array}{c}42 \\
(37 \%)\end{array}$ & $\begin{array}{c}71 \\
(63 \%)\end{array}$ & $\begin{array}{c}71 \\
(48 \%)\end{array}$ & $\begin{array}{c}78 \\
(52 \%)\end{array}$ & $\begin{array}{c}10 \\
(50 \%)\end{array}$ & $\begin{array}{c}10 \\
(50 \%)\end{array}$ & $\begin{array}{c}14 \\
(37 \%)\end{array}$ & $\begin{array}{c}24 \\
(63 \%)\end{array}$ \\
\hline 2004-2008 & $\begin{array}{c}51 \\
(57 \%)\end{array}$ & $\begin{array}{c}39 \\
(43 \%)\end{array}$ & $\begin{array}{c}58 \\
(46 \%)\end{array}$ & $\begin{array}{c}68 \\
(54 \%)\end{array}$ & $\begin{array}{c}80 \\
(44 \%)\end{array}$ & $\begin{array}{c}100 \\
(56 \%)\end{array}$ & $\begin{array}{c}13 \\
(65 \%)\end{array}$ & $\begin{array}{c}7 \\
(35 \%)\end{array}$ & $\begin{array}{c}20 \\
(43 \%)\end{array}$ & $\begin{array}{c}27 \\
(57 \%)\end{array}$ \\
\hline \multicolumn{11}{|l|}{ Lung cancer type: } \\
\hline Squamous cell carcinoma & $\begin{array}{c}25 \\
(20 \%)\end{array}$ & $\begin{array}{c}69 \\
(42 \%)\end{array}$ & $\begin{array}{c}29 \\
(21 \%)\end{array}$ & $\begin{array}{c}107 \\
(38 \%)\end{array}$ & $\begin{array}{c}28 \\
(13 \%)\end{array}$ & $\begin{array}{c}87 \\
(29 \%)\end{array}$ & & & & \\
\hline Adenocarcinoma & $\begin{array}{c}71 \\
(57 \%)\end{array}$ & $\begin{array}{c}57 \\
(35 \%)\end{array}$ & $\begin{array}{c}79 \\
(56 \%)\end{array}$ & $\begin{array}{c}109 \\
(39 \%)\end{array}$ & $\begin{array}{c}147 \\
(66 \%)\end{array}$ & $\begin{array}{c}143 \\
(48 \%)\end{array}$ & & & & \\
\hline Other lung cancer ${ }^{\mathrm{a}}$ & $\begin{array}{c}12 \\
(10 \%)\end{array}$ & $\begin{array}{c}22 \\
(13 \%)\end{array}$ & $\begin{array}{c}20 \\
(14 \%)\end{array}$ & $\begin{array}{c}34 \\
(12 \%)\end{array}$ & $\begin{array}{c}30 \\
(14 \%)\end{array}$ & $\begin{array}{c}43 \\
(15 \%)\end{array}$ & & & & \\
\hline Carcinoid & $\begin{array}{c}12 \\
(10 \%)\end{array}$ & $\begin{array}{c}5 \\
(3 \%)\end{array}$ & $\begin{array}{c}3 \\
(2 \%)\end{array}$ & 0 & 1 & 0 & & & & \\
\hline Clinical lung cancer & $\begin{array}{c}4 \\
(3 \%)\end{array}$ & $\begin{array}{c}12 \\
(7 \%)\end{array}$ & $\begin{array}{c}10 \\
(7 \%)\end{array}$ & $\begin{array}{c}31 \\
(11 \%)\end{array}$ & $\begin{array}{c}16 \\
(7 \%)\end{array}$ & $\begin{array}{c}25 \\
(8 \%)\end{array}$ & & & & \\
\hline \multicolumn{11}{|l|}{ Primary therapy: } \\
\hline Surgery $\pm \mathrm{RT}^{\mathrm{b}} \pm$ Chemo $^{\mathrm{c}}$ & $\begin{array}{c}99 \\
(80 \%)\end{array}$ & $\begin{array}{c}119 \\
(72 \%)\end{array}$ & $\begin{array}{c}32 \\
(23 \%)\end{array}$ & $\begin{array}{c}40 \\
(14 \%)\end{array}$ & 2 & 1 & $\begin{array}{c}4 \\
(8 \%)\end{array}$ & $\begin{array}{c}5 \\
(9 \%)\end{array}$ & & \\
\hline Curative RT \pm Chemo & $\begin{array}{c}9 \\
(7 \%)\end{array}$ & $\begin{array}{c}14 \\
(9 \%)\end{array}$ & $\begin{array}{c}26 \\
(18 \%)\end{array}$ & $\begin{array}{c}47 \\
(17 \%)\end{array}$ & & & 34 & 38 & & \\
\hline Palliative RT \pm Chemo & 5 & 10 & $\begin{array}{c}24 \\
(17 \%)\end{array}$ & $\begin{array}{c}66 \\
(24 \%)\end{array}$ & $\begin{array}{c}39 \\
(18 \%)\end{array}$ & $\begin{array}{c}56 \\
(19 \%)\end{array}$ & $(69 \%)^{\mathrm{d}}$ & $(64 \%)^{d}$ & $\begin{array}{c}3 \\
(5 \%)\end{array}$ & $\begin{array}{c}2 \\
(2 \%)\end{array}$ \\
\hline Chemo \pm palliative RT & $(4 \%)^{e}$ & $(6 \%)^{e}$ & $\begin{array}{c}28 \\
(20 \%)\end{array}$ & $\begin{array}{c}40 \\
(14 \%)\end{array}$ & $\begin{array}{c}107 \\
(48 \%)\end{array}$ & $\begin{array}{c}120 \\
(40 \%)\end{array}$ & $\begin{array}{c}9 \\
(18 \%)\end{array}$ & $\begin{array}{c}14 \\
(24 \%)\end{array}$ & $\begin{array}{c}47 \\
(84 \%)\end{array}$ & $\begin{array}{c}76 \\
(75 \%)\end{array}$ \\
\hline No therapy & $\begin{array}{c}11 \\
(9 \%)\end{array}$ & $\begin{array}{c}22 \\
(13 \%)\end{array}$ & $\begin{array}{c}31 \\
(22 \%)\end{array}$ & $\begin{array}{c}88 \\
(31 \%)\end{array}$ & $\begin{array}{c}74 \\
(33 \%)\end{array}$ & $\begin{array}{c}121 \\
(41 \%)\end{array}$ & $\begin{array}{c}2 \\
(4 \%)\end{array}$ & $\begin{array}{c}2 \\
(3 \%)\end{array}$ & $\begin{array}{c}6 \\
(11 \%)\end{array}$ & $\begin{array}{c}24 \\
(23 \%)\end{array}$ \\
\hline
\end{tabular}

${ }^{\mathrm{a}}$ Other lung cancer includes large cell—adenosquamous and adenocystic cancer. ${ }^{\mathrm{b}} \mathrm{RT}$; radiotherapy, ${ }^{\mathrm{c}}$ Chemo; chemotherapy. ${ }^{\mathrm{d}}$ Chemotherapy + radiotherapy. ${ }^{e}$ Palliative radiotherapy \pm chemotherapy or chemotherapy. 


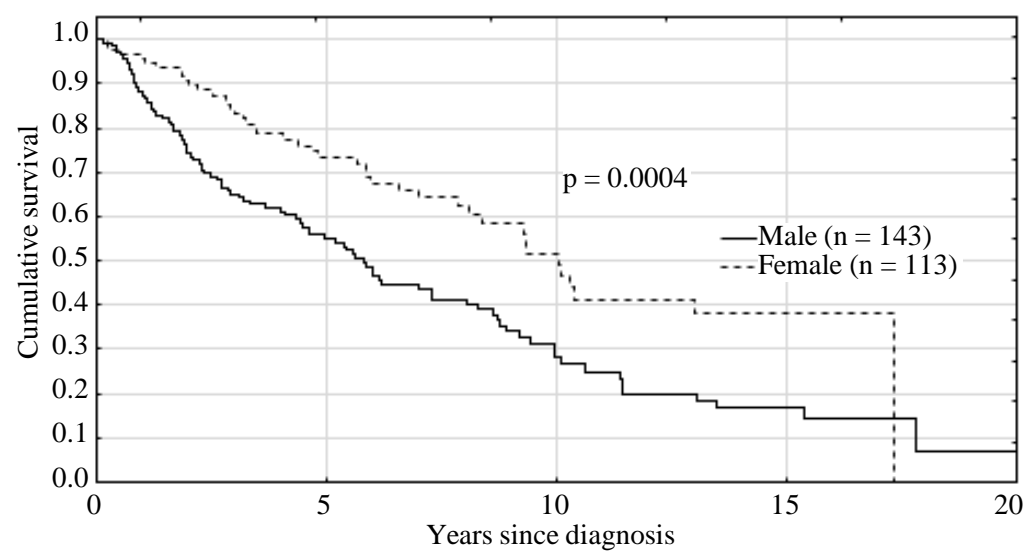

(a)

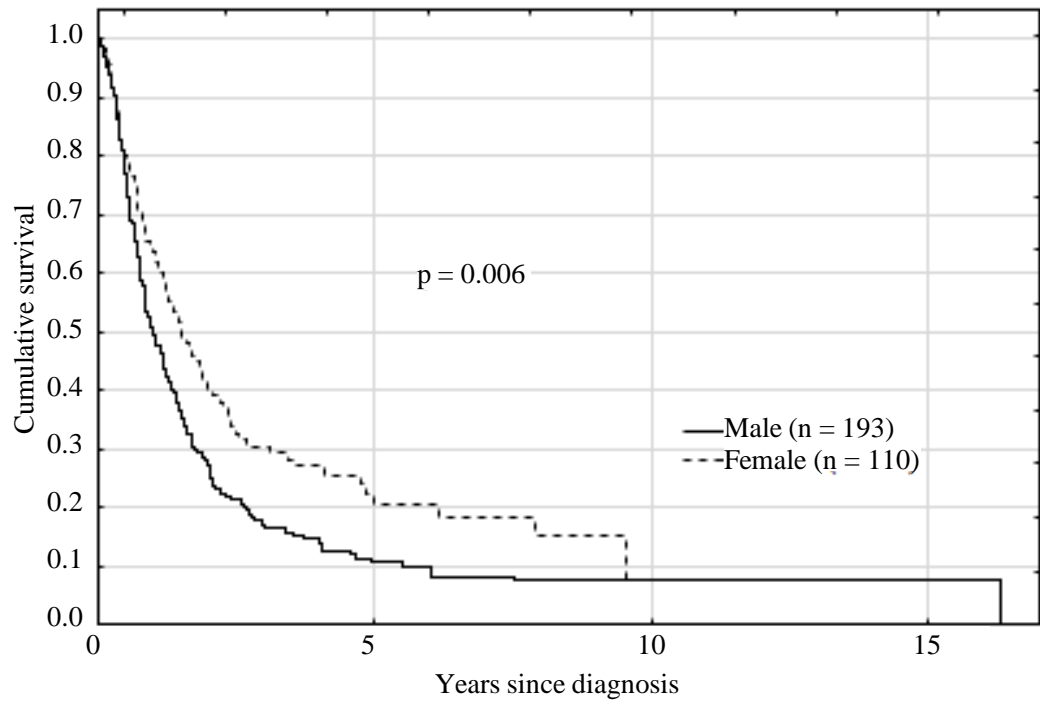

(b)

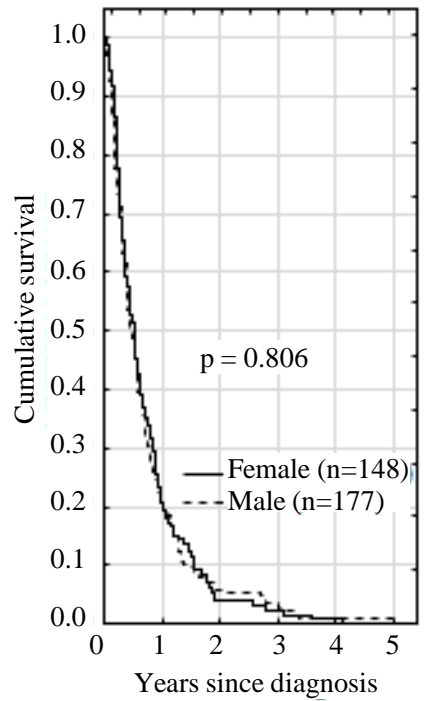

(c)

Figure 2. Gender-related survival in patients with NSCLC [(a) stages 1-2, (b) stage 3, (c) stage 4] with active therapy (surgery and/or radiotherapy and/or chemotherapy) in eastern Scania from 1989-2008.

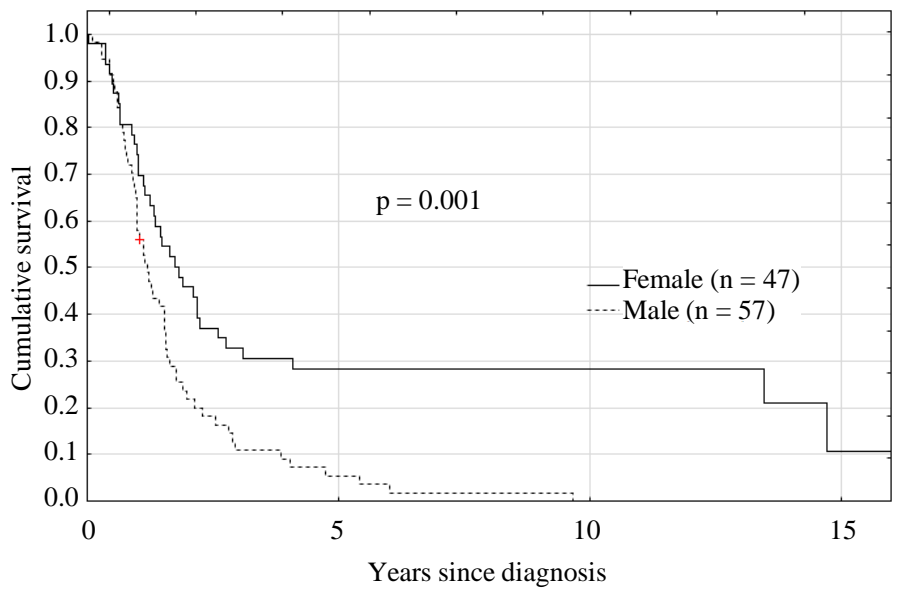

(a)

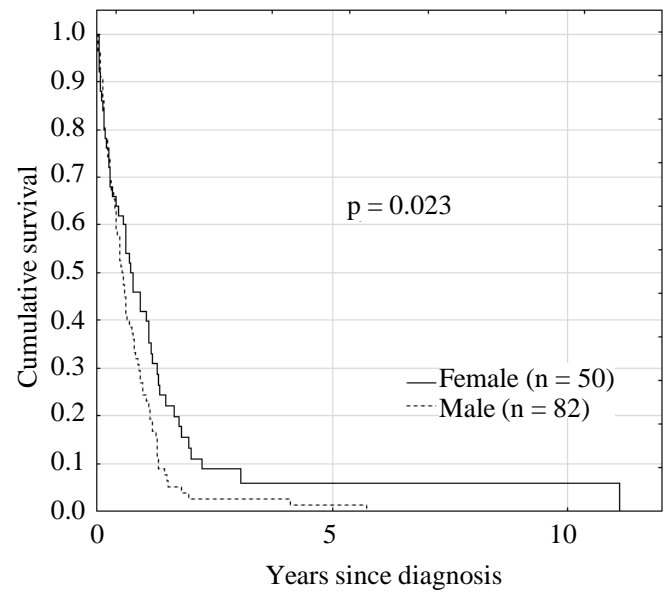

(b)

Figure 3. Gender-related survival in patients with SCLC [(a) limited disease, (b) extensive disease], with active therapy (chemotherapy and/or radiotherapy and/or surgery) in eastern Scania from 1989-2008. 
Table 2. Univariate- and multivariate analyses of prognostic factors for survival in NSCLC.

\begin{tabular}{|c|c|c|c|c|c|c|}
\hline \multirow[b]{2}{*}{ Stage $1+2:^{a}$} & \multicolumn{3}{|c|}{ Univariate } & \multicolumn{3}{|c|}{ Multivariate } \\
\hline & HR & $95 \%$ CI & $\mathrm{P}$ & HR & $95 \%$ CI & $\mathrm{P}$ \\
\hline \multicolumn{7}{|l|}{ Gender } \\
\hline Female $(n=124)$ & 1.00 & ref & & 1.00 & ref & \\
\hline Male $(n=165)$ & 1.88 & $1.35-2.60$ & 0.0002 & 1.71 & $1.21-2.43$ & 0.002 \\
\hline \multicolumn{7}{|l|}{ Age } \\
\hline$<70(\mathrm{n}=135)$ & 1.00 & ref & & 1.00 & ref & \\
\hline$\geq 70(\mathrm{n}=154)$ & 2.75 & $1.98-3.82$ & $<0.001$ & 1.69 & $1.14-2.49$ & 0.009 \\
\hline \multicolumn{7}{|l|}{ Performance status } \\
\hline $0-1(n=233)$ & 1.00 & ref & & 1.00 & ref & \\
\hline $2-4(n=56)$ & 6.06 & $4.17-8.81$ & $<0.001$ & 2.29 & $1.40-3.74$ & 0.0009 \\
\hline \multicolumn{7}{|l|}{ Stage $3:^{b}$} \\
\hline \multicolumn{7}{|l|}{ Gender } \\
\hline Female $(\mathrm{n}=141)$ & 1.00 & ref & & 1.00 & ref & \\
\hline Male $(n=281)$ & 1.42 & $1.14-1.77$ & 0.002 & 1.33 & $1.05-1.69$ & 0.017 \\
\hline \multicolumn{7}{|l|}{ Age } \\
\hline$<70(\mathrm{n}=177)$ & 1.00 & ref & & 1.00 & ref & \\
\hline$\geq 70(\mathrm{n}=245)$ & 1.53 & $1.24-1.89$ & $<0.001$ & 0.85 & $0.67-1.08$ & 0.188 \\
\hline \multicolumn{7}{|l|}{ Performance status } \\
\hline $0-1(\mathrm{n}=239)$ & 1.00 & ref & & 1.00 & ref & \\
\hline $2-4(n=183)$ & 3.52 & $2.83-4.39$ & $<0.001$ & 2.06 & $1.60-2.65$ & $<0.001$ \\
\hline \multicolumn{7}{|l|}{ Stage $4:^{\mathrm{C}}$} \\
\hline \multicolumn{7}{|l|}{ Gender } \\
\hline Female (n = 222) & 1.00 & ref & & 1.00 & ref & \\
\hline Male $(n=298)$ & 1.09 & $0.91-1.30$ & 0.350 & 1.00 & $0.83-1.20$ & 0.968 \\
\hline \multicolumn{7}{|l|}{ Age } \\
\hline$<70(\mathrm{n}=279)$ & 1.00 & ref & & 1.00 & ref & \\
\hline$\geq 70(\mathrm{n}=241)$ & 1.20 & $1.01-1.43$ & 0.042 & 0.68 & $0.56-0.83$ & 0.0002 \\
\hline \multicolumn{7}{|l|}{ Performance status } \\
\hline $0-1(n=167)$ & 1.00 & ref & & 1.00 & ref & \\
\hline $2-4(n=353)$ & 3.19 & $2.61-3.88$ & $<0.001$ & 2.80 & $2.25-3.47$ & $<0.001$ \\
\hline
\end{tabular}

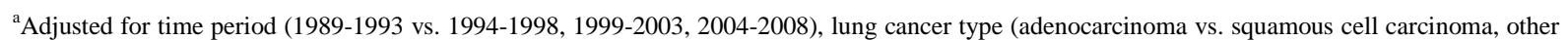
cancer, carcinoid, clinical cancer) and primary therapy (surgery \pm radiotherapy \pm chemotherapy vs. curative radiotherapy \pm chemotherapy, palliative radiotherapy \pm /or chemotherapy, no therapy). ${ }^{b}$ Adjusted for time period (see stage $1+2$ ), lung cancer type (see stage $1+2$ ) and primary therapy (surgery \pm radiotherapy \pm chemotherapy vs. curative radiotherapy \pm chemotherapy, palliative radiotherapy \pm chemotherapy, chemotherapy, no therapy). ${ }^{c}$ Adjusted for time period (see stage $1+2$ ), lung cancer type (see stage $1+2$ ) and primary therapy (chemotherapy vs. chemotherapy + palliative radiotherapy, palliative radiotherapy, surgery, no therapy)

stage 1-2. However, in NSCLC stage 4 and SCLC stage ED, age $\geq 70$ had a decreased HR for death.

Non-surgical therapy (radiotherapy \pm chemotherapy) had an increased HR for death in NSCLC stages 1-3, but this was not significant for curative radiotherapy in stages 1-2.

Lung cancer survival has only improved the last time periods 1999-2008 (compared to 1989-1993) in NSCLC stages $1-2$. 
Table 3. Univariate- and multivariate analyses of prognostic factors for survival in SCLC.

\begin{tabular}{|c|c|c|c|c|c|c|}
\hline \multirow[b]{2}{*}{ Limited disease: ${ }^{\mathrm{a}}$} & \multicolumn{3}{|c|}{ Univariate } & \multicolumn{3}{|c|}{ Multivariate } \\
\hline & HR & $95 \%$ CI & $\mathrm{P}$ & HR & $95 \%$ CI & $\mathrm{P}$ \\
\hline \multicolumn{7}{|l|}{ Gender } \\
\hline Female $(n=49)$ & 1.00 & ref & & 1.00 & ref & \\
\hline Male $(\mathrm{n}=59)$ & 2.10 & $1.37-3.22$ & 0.0007 & 2.43 & $1.45-4.06$ & 0.0007 \\
\hline \multicolumn{7}{|l|}{ Age } \\
\hline$<70(\mathrm{n}=68)$ & 1.00 & ref & & 1.00 & ref & \\
\hline$\geq 70(\mathrm{n}=40)$ & 1.64 & $1.08-2.48$ & 0.020 & 1.07 & $0.64-1.77$ & 0.804 \\
\hline \multicolumn{7}{|l|}{ Performance status } \\
\hline $0-1(\mathrm{n}=74)$ & 1.00 & ref & & 1.00 & ref & \\
\hline $2-4(n=34)$ & 2.01 & $1.29-3.11$ & 0.002 & 2.18 & $1.17-4.06$ & 0.014 \\
\hline \multicolumn{7}{|l|}{ Extensive disease: ${ }^{\mathbf{b}}$} \\
\hline \multicolumn{7}{|l|}{ Gender } \\
\hline Female $(\mathrm{n}=56)$ & 1.00 & ref & & 1.00 & ref & \\
\hline Male $(n=102)$ & 1.62 & $1.15-2.28$ & 0.006 & 1.59 & $1.12-2.26$ & 0.010 \\
\hline \multicolumn{7}{|l|}{ Age } \\
\hline$<70(\mathrm{n}=80)$ & 1.00 & ref & & 1.00 & ref & \\
\hline$\geq 70(\mathrm{n}=78)$ & 1.28 & $0.93-1.77$ & 0.133 & 0.67 & $0.45-0.99$ & 0.046 \\
\hline \multicolumn{7}{|l|}{ Performance status } \\
\hline $0-1(n=42)$ & 1.00 & ref & & 1.00 & ref & \\
\hline $2-4(n=116)$ & 2.42 & $1.67-3.50$ & $<0.001$ & 2.48 & $1.63-3.77$ & $<0.001$ \\
\hline
\end{tabular}

${ }^{a}$ Adjusted for time periods (see Table 2) and primary therapy (chemotherapy + radiotherapy vs. surgery + chemotherapy, chemotherapy, no therapy).

${ }^{\mathrm{b}}$ Adjusted for time periods (see Table 2) and primary therapy (chemotherapy vs. chemotherapy + radiotherapy, radiotherapy, no therapy).

\subsection{Gender, Age and Survival in 246 Patients Not in Local Registry}

Of the 212 NSCLC patients not referred to Central Hospital 35\% were females (39\% in registry) and median age was 70 years in females (68 years in registry) and 74 years in males (71 years in registry). In the 34 SCLC patients not referred 35\% were females (39\% in registry) and median age was 66 years in females (65 years in registry) and 68 years in males (70 years in registry).

Lung cancer diagnoses were performed at autopsy in 18.3\% (45/246) of the patients not referred to our hospital.

In the 212 patients with NSCLC not referred to Central Hospital, Kristianstad, the 5-year survival was $10.8 \%$ and $4.3 \%$ in females and in males, respectively $(\mathrm{p}=0.003)$. Of the 34 patients with SCLC who were not referred to our clinic, none survived for 5 years.

\section{Discussion}

\subsection{NSCLC}

One of the main findings in this study was the increased survival in females with NSCLC in stages 1-3, but not in stage 4. DeCamp Jr. reported that in completely resected stage 1 patients, males had a worse prognosis than females [6]. This finding was confirmed by Ferguson et al. [14]. Female patients who underwent surgery for 
NSCLC had better survival rates than males in studies by de Perrot et al. [15]. They also reported better female survival in the adenocarcinoma subgroup corresponding to our study. Alexio et al. found female survival advantage in resected patients with adenocarcinoma and squamous cell carcinoma, but the female survival advantage was significantly better only in stage 1 [16]. Batevik et al. reported better survival in operated female patients but did not report any effect of histology [17]. Cerfolio et al. reported that the 5-year survival rate of women with pathological stages 1-3 was better than in men [18]. In our study, resected female patients in stages 1-3 had significantly better survival than males.

McGovern et al. reported a worse prognosis for male compared to female stages 1-3 patients who were treated with radiotherapy [19], which is consistent with our finding of better female survival in stage 3 patients treated with radiotherapy.

Wheatley-Price et al. reported longer overall survival in females compared to males who were treated in five pooled chemotherapy trials [20]. In subgroup analysis the female survival advantage was significant only in adenocarcinoma patients. In our patients with stage 4 there were no survival difference between females and males, nor in the subgroup of adenocarcinoma.

The reason for the better survival in females with early stages of NSCLC could be a higher frequency of more highly differentiated adenocarcinomas with low proliferation indexes as reported by Sterlacci et al. [21]. Female patients with squamous cell carcinoma had better survival than males only in stage 3 . This could be due to the heterogeneous stage 3, but a non-significantly higher surgery rate in females (31\%) compared to males (24\%) can influence. A somewhat higher frequency of carcinoids in females could contribute to the better survival in females in our study, but this factor was adjusted for in the multivariate analyses. More male patients could have been former or current smokers, which could contribute to more comorbidities in male patients.

Patient performance status was an important prognostic factor in all stages of NSCLC, which is concordant with studies by Sculier et al. [22] and Kawaguchi et al. [23].

Age $\geq 70$ years was a negative prognostic factor only in NSCLC stages 1-2 patients, which is in line with a study by Pagano et al., who reported age $\geq 75$ in early stages as a negative prognostic factor [24].

We found that patients with stage 4 and aged $\geq 70$ years had a better prognosis than patients aged $<70$ years. This has been previously reported by Albain et al. [25]. In later studies, there were no differences in outcome in patients younger than age $<70$ compared to those who were at least 70 years old [26].

\subsection{SCLC}

Another main finding in this study was the increased survival in females with SCLC both in cases of LD and ED. In the literature, these results have not been consistent. In 1988, Johnson et al. reported an improved 2.5-year survival in females with SCLC compared to males in North America [5]. Trials in Cancer and Leukaemia Group B reported improved survival in females in cases of both LD as well as ED [27]. Albain et al. found that female gender was a good prognostic factor only in patients with LD in the Southwest Oncology Group trials [7]. In Europe, Wolf et al. reported the increased survival of females with LD and ED only in ages $<60$ years [28]. In a population-based study in France, Mennecier et al. reported no survival difference between genders [29]. Jansen-Heijnen et al. of the Netherlands reported the increased survival of females, but only in LD cases [30]. In trials from the UK, Wheatley-Price et al. reported the decreased HR for female patients with LD [31].

Our findings of better survival in female patients with both LD and ED have, to our knowledge, not been previously reported in a European population study. However, in a recent and very large (42,000 males, 38,000 females) United States national cancer database study from 1992-2007, Gaspar et al. reported the increased HR for death in males as compared to females in both LD (HR 1.19) and ED (HR 1.13) patients [32]. In that report, initial staging was conducted according to the TNM system, in which patients with malignant pleural fluid (stage $3 \mathrm{~b}$ ) were included in the limited disease group. The study by Gaspar et al. reported the increased HR for death in patients aged $\geq 70$ years in both LD and ED patients. In our study, there was no such age difference, but rather decreased HR for death in patients with $E D$ and age $\geq 70$ years. One reason could be that our study also included performance status, and the increased HR for age $\geq 70$ years in the univariate analyses changed when adjusted for performance status. Our study also demonstrated that a performance status $>1$ in both the LD and ED patients is a strong prognostic factor and should be considered with greater emphasis than the factor of age $\geq$ 70.

Patients with LD who were treated with surgery + chemotherapy had significantly better survival than patients treated with chemoradiation. This finding is in agreement with the findings of the Gaspar database study. How- 
ever, in our study the reduced HR 0.48 (95\% CI 0.20 - 1.13) compared to chemoradiation was not significant due to the few patients who underwent surgery.

The reasons for the better prognosis in female patients with SCLC are still unknown. Increased toxicity as well as a better response to chemotherapy in females [33] have been suggested to be associated with reduced drug clearance due to lower levels of p-glycoprotein in females [34].

The female survival advantage in lung cancer can also be related to a general better female survival in most cancer sites as reported in Eurocare-4 [35].

Thyroid Transcription Factor-1 (TTF-1) is expressed in adenocarcinoma and is correlated with better prognosis. It is more frequently expressed in females than in males [36]. A Chinese study reports expression of TTF-1 in SCLC and they also found better prognosis with TTF-1 expression [37].

\subsection{Limitations and Strengths}

The present study includes patients who were diagnosed and treated in the hospital according to contemporary guidelines. Treatment recommendations have also changed over the 20-year study period.

The diagnosis date was set at the pathologic reply date because of a rather long reply delay during the early 1990s.

Of all of the lung cancer patients in eastern Scania, 14\% were not referred to our hospital; Of these, $18.3 \%$ were diagnosed by autopsy findings. The remaining patients were possibly clinically advanced with poor general health and were, therefore, not referred. This possibility is supported by the rather short survival rate of patients not in the registry.

Our local registry did not contain a pathological staging system, and only patients primarily undergoing surgery were assigned a pathology stage. In the remaining patients, the stages were based on clinical findings.

Most of the patients with lung cancer in the 1980s were smokers or former smokers, so for this reason we omitted this information from the registry.

The pathology reports have not been reviewed, and variations over time between different pathologists with varied histochemical techniques cannot be ruled out as influential factors in this study.

Eastern Scania is a well-defined area that has exhibited a rather small variation in population structure over the 20 years this study covers.

The extended time period of two decades with only one person involved in the diagnosis and/or treatment of almost all patients, as well as performing all registrations in exactly the same way, minimized the risk of judgement discrepancies.

\section{Conclusion}

The present population based study strengthens earlier reports of a female survival advantage in NSCLC stages 1-3, but not in metastatic stage 4, and this is also demonstrated for the adenocarcinoma subgroup. The study also confirms the better prognosis in females with SCLC, especially in patients with LD, but also significantly in patients with ED. The study also demonstrates the importance of patient performance status as an independent prognostic factor in all tumour stages. Age $\geq 70$ years was only a negative prognostic factor in early stages of NSCLC.

\section{Acknowledgements}

This research project was financially supported by the Medical Research Council, Central Hospital, Kristianstad and Kristianstad University.

\section{References}

[1] Mountain, C.F. (1997) Revisions in the International System for Staging Lung Cancer. Chest, 111, 1710-1717. http://dx.doi.org/10.1378/chest.111.6.1710

[2] Buccheri, G., Ferrigno, D. and Tamburini, M. (1996) Karnofsky and ECOG Performance Status Scoring in Lung Cancer: A Prospective, Longitudinal Study of 536 Patients from a Single Institution. European Journal of Cancer, 32A, 1135-1141. http://dx.doi.org/10.1016/0959-8049(95)00664-8

[3] Green, R.A., Humphrey, E., Close, H. and Patno, M.E. (1969) Alkylating Agents in Bronchogenic Carcinoma. The 
American Journal of Medicine, 46, 516-525. http://dx.doi.org/10.1016/0002-9343(69)90071-0

[4] Tammemagi, C.M., Neslund-Dudas, C., Simoff, M. and Kvale, P. (2004) In Lung Cancer Patients, Age, Race-Ethnicity, Gender and Smoking Predict Adverse Comorbidity, Which in Turn Predicts Treatment and Survival. Journal of Clinical Epidemiology, 57, 597-609. http://dx.doi.org/10.1016/j.jclinepi.2003.11.002

[5] Johnson, B.E., Steinberg, S.M., Phelps, R., Edison, M., Veach, S.R. and Ihde, D.C. (1988) Female Patients with Small Cell Lung Cancer Live Longer than Male Patients. The American Journal of Medicine, 85, 194-196. http://dx.doi.org/10.1016/S0002-9343(88)80341-3

[6] DeCamp Jr., M.M., Strauss, G.M., Kwiatkowski, D.J., Harpole, D., Godleski, J., Shieh, D., et al. (1997) 731 Gender Predicts Survival in Stage I NSCLC: Clinico-Pathologic and Molecular Analysis of 250 Patients. Lung Cancer, 18, 187-188.

[7] Albain, K.S., Crowley, J.J., LeBlanc, M. and Livingston, R.B. (1990) Determinants of Improved Outcome in SmallCell Lung Cancer: An Analysis of the 2580-Patient Southwest Oncology Group Data Base. Journal of Clinical Oncology, 8, 1563-1574.

[8] Svensson, G., Ewers, S.-B., Ohlsson, O. and Olsson, H. (2013) Prognostic Factors in Lung Cancer in a Defined Geographical Area over Two Decades with a Special Emphasis on Gender. The Clinical Respiratory Journal, 7, 91-100. http://dx.doi.org/10.1111/j.1752-699X.2012.00289.x

[9] Statistiska Centralbyrån (The Central Bureau of Statistics, S.: Statistiska Centralbyrån (the Central Bureau of Statistics, Sweden).

[10] Organization, W.H. (1981) Histological Typing of Lung Tumours. 2nd Edition, World Health Organization, Geneva.

[11] Mountain, C.F. (1986) A New International Staging System for Lung Cancer. Chest, 89, 225S-233S. http://dx.doi.org/10.1378/chest.89.4_Supplement.225S

[12] Zelen, M. (1973) Keynote Address on Biostatistics and Data Retrieval. Cancer Chemotherapy Reports, 4, 31-42.

[13] Oken, M.M., Creech, R.H., Tormey, D.C., Horton, J., Davis, T.E., McFadden, E.T., et al. (1982) Toxicity and Response Criteria of the Eastern Cooperative Oncology Group. American Journal of Clinical Oncology, 5, 649-655. http://dx.doi.org/10.1097/00000421-198212000-00014

[14] Ferguson, M.K., Wang, J., Hoffman, P.C., Haraf, D.J., Olak, J., Masters, G.A., et al. (2000) Sex-Associated Differences in Survival of Patients Undergoing Resection for Lung Cancer. The Annals of Thoracic Surgery, 69, 245-249; Discussion 249-250. http://dx.doi.org/10.1016/S0003-4975(99)01078-4

[15] de Perrot, M., Licker, M., Bouchardy, C., Usel, M., Robert, J. and Spiliopoulos, A. (2000) Sex Differences in Presentation, Management and Prognosis of Patients with Non-Small Cell Lung Carcinoma. The Journal of Thoracic and Cardiovascular Surgery, 119, 21-26. http://dx.doi.org/10.1016/S0022-5223(00)70213-3

[16] Alexiou, C., Patrick Onyeaka, C.V., Beggs, D., Akar, R., Beggs, L., Salama, F.D., et al. (2002) Do Women Live Longer Following Lung Resection for Carcinoma? European Journal of Cardio-Thoracic Surgery, 21, 319-325. http://dx.doi.org/10.1016/S1010-7940(01)01114-9

[17] Batevik, R., Grong, K., Segadal, L. and Stangeland, L. (2005) The Female Gender Has a Positive Effect on Survival Independent of Background Life Expectancy Following Surgical Resection of Primary Non-Small Cell Lung Cancer: A Study of Absolute and Relative Survival over 15 Years. Lung Cancer, 47, 173-181. http://dx.doi.org/10.1016/j.lungcan.2004.08.014

[18] Cerfolio, R.J., Bryant, A.S., Scott, E., Sharma, M., Robert, F., Spencer, S.A., et al. (2006) Women with Pathologic Stage I, II and III Non-Small Cell Lung Cancer Have Better Survival than Men. Chest, 130, 1796-1802. http://dx.doi.org/10.1378/chest.130.6.1796

[19] McGovern, S.L., Liao, Z.X., Bucci, M.K., McAleer, M.F., Jeter, M.D., Chang, J.Y., et al. (2009) Is Sex Associated with the Outcome of Patients Treated with Radiation for Nonsmall Cell Lung Cancer? Cancer, 115, 3233-3242. http://dx.doi.org/10.1002/cncr.24361

[20] Wheatley-Price, P., Blackhall, F., Lee, S.M., Ma, C., Ashcroft, L., Jitlal, M., et al. (2010) The Influence of Sex and Histology on Outcomes in Non-Small-Cell Lung Cancer: A Pooled Analysis of Five Randomized Trials. Annals of Oncology: Official Journal of the European Society for Medical Oncology/ESMO, 21, 2023-2028.

[21] Sterlacci, W., Tzankov, A., Veits, L., Oberaigner, W., Schmid, T., Hilbe, W., et al. (2011) The Prognostic Impact of Sex on Surgically Resected Non-Small Cell Lung Cancer Depends on Clinicopathologic Characteristics. American Journal of Clinical Pathology, 135, 611-618. http://dx.doi.org/10.1309/AJCPQF24NYWNMVMG

[22] Sculier, J.P., Chansky, K., Crowley, J.J., Van Meerbeeck, J. and Goldstraw, P. (2008) The Impact of Additional Prognostic Factors on Survival and Their Relationship with the Anatomical Extent of Disease Expressed by the 6th Edition of the TNM Classification of Malignant Tumors and the Proposals for the 7th Edition. Journal of Thoracic Oncology: Official Publication of the International Association for the Study of Lung Cancer, 3, 457-466. 
[23] Kawaguchi, T., Takada, M., Kubo, A., Matsumura, A., Fukai, S., Tamura, A., et al. (2010) Performance Status and Smoking Status Are Independent Favorable Prognostic Factors for Survival in Non-Small Cell Lung Cancer: A Comprehensive Analysis of 26,957 Patients with NSCLC. Journal of Thoracic Oncology: Official Publication of the International Association for the Study of Lung Cancer, 5, 620-630.

[24] Pagano, E., Filippini, C., Di Cuonzo, D., Ruffini, E., Zanetti, R., Rosso, S., et al. (2010) Factors Affecting Pattern of Care and Survival in a Population-Based Cohort of Non-Small-Cell Lung Cancer Incident Cases. Cancer Epidemiology, 34, 483-489. http://dx.doi.org/10.1016/j.canep.2010.04.002

[25] Albain, K.S., Crowley, J.J., LeBlanc, M. and Livingston, R.B. (1991) Survival Determinants in Extensive-Stage NonSmall-Cell Lung Cancer: The Southwest Oncology Group Experience. Journal of Clinical Oncology, 9, 1618-1626.

[26] Foegle, J., Hedelin, G., Lebitasy, M.P., Purohit, A., Velten, M. and Quoix, E. (2005) Non-Small-Cell Lung Cancer in a French Department (1982-1997): Management and Outcome. British Journal of Cancer, 92, 459-466.

[27] Spiegelman, D., Maurer, L.H., Ware, J.H., Perry, M.C., Chahinian, A.P., Comis, R., et al. (1989) Prognostic Factors in Small-Cell Carcinoma of the Lung: An Analysis of 1,521 Patients. Journal of Clinical Oncology: Official Journal of the American Society of Clinical Oncology, 7, 344-354.

[28] Wolf, M., Holle, R., Hans, K., Drings, P. and Havemann, K. (1991) Analysis of Prognostic Factors in 766 Patients with Small Cell Lung Cancer (SCLC): The Role of Sex as a Predictor for Survival. British Journal of Cancer, 63, 986-992. http://dx.doi.org/10.1038/bjc.1991.215

[29] Mennecier, B., Lebitasy, M.-P., Moreau, L., Hedelin, G., Purohit, A., Galichet, C., et al. (2003) Women and Small Cell Lung Cancer: Social Characteristics, Medical History, Management and Survival: A Retrospective Study of All the Male and Female Cases Diagnosed in Bas-Rhin (Eastern France) between 1981 and 1994. Lung Cancer, 42, 141-152. http://dx.doi.org/10.1016/S0169-5002(03)00284-8

[30] Janssen-Heijnen, M.L.G., Lemmens, V.E.P.P., van den Borne, B.E.E.M., Biesma, B., Oei, S.B. and Coebergh, J.W.W. (2007) Negligible Influence of Comorbidity on Prognosis of Patients with Small Cell Lung Cancer: A PopulationBased Study in the Netherlands. Critical Reviews in Oncology/Hematology, 62, 172-178. http://dx.doi.org/10.1016/j.critrevonc.2006.11.005

[31] Wheatley-Price, P., Ma, C., Ashcroft, L.F., Nankivell, M., Stephens, R.J., White, S.C., et al. (2010) The Strength of Female Sex as a Prognostic Factor in Small-Cell Lung Cancer: A Pooled Analysis of Chemotherapy Trials from the Manchester Lung Group and Medical Research Council Clinical Trials Unit. Annals of Oncology: Official Journal of the European Society for Medical Oncology/ESMO, 21, 232-237.

[32] Gaspar, L.E., McNamara, E.J., Gay, E.G., Putnam, J.B., Crawford, J., Herbst, R.S., et al. (2012) Small-Cell Lung Cancer: Prognostic Factors and Changing Treatment over 15 Years. Clinical Lung Cancer, 13, 115-122. http://dx.doi.org/10.1016/j.cllc.2011.05.008

[33] Singh, S., Parulekar, W., Murray, N., Feld, R., Evans, W.K., Tu, D., et al. (2005) Influence of Sex on Toxicity and Treatment Outcome in Small-Cell Lung Cancer. Journal of Clinical Oncology, 23, 850-856. http://dx.doi.org/10.1200/JCO.2005.03.171

[34] Davis, M. (2005) Gender Differences in p-Glycoprotein: Drug Toxicity and Response. Journal of Clinical Oncology, 23, 6439-6440. http://dx.doi.org/10.1200/JCO.2005.01.9232

[35] Micheli, A., Ciampichini, R., Oberaigner, W., Ciccolallo, L., de Vries, E., Izarzugaza, I., et al. (2009) The Advantage of Women in Cancer Survival: An Analysis of EUROCARE-4 Data. European Journal of Cancer, 45, 1017-1027. http://dx.doi.org/10.1016/j.ejca.2008.11.008

[36] Anagnostou, V.K., Syrigos, K.N., Bepler, G., Homer, R.J. and Rimm, D.L. (2009) Thyroid Transcription Factor 1 Is an Independent Prognostic Factor for Patients With Stage I Lung Adenocarcinoma. Journal of Clinical Oncology, 27, 271-278. http://dx.doi.org/10.1200/JCO.2008.17.0043

[37] Lv, X., Sun, L., Zhan, Z., Sun, B. and Wang, C. (2012) Expression and Prognostic Value of TTF-1 and CK in Small Cell Lung Carcinoma. Chinese Journal of Clinical Oncology, 39, 273-277. 
Scientific Research Publishing (SCIRP) is one of the largest Open Access journal publishers. It is currently publishing more than 200 open access, online, peer-reviewed journals covering a wide range of academic disciplines. SCIRP serves the worldwide academic communities and contributes to the progress and application of science with its publication.

Other selected journals from SCIRP are listed as below. Submit your manuscript to us via either submit@scirp.org or Online Submission Portal.
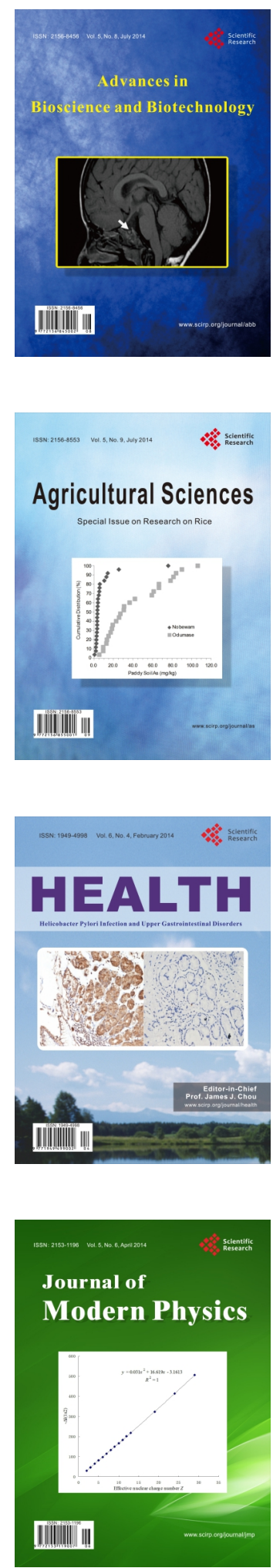
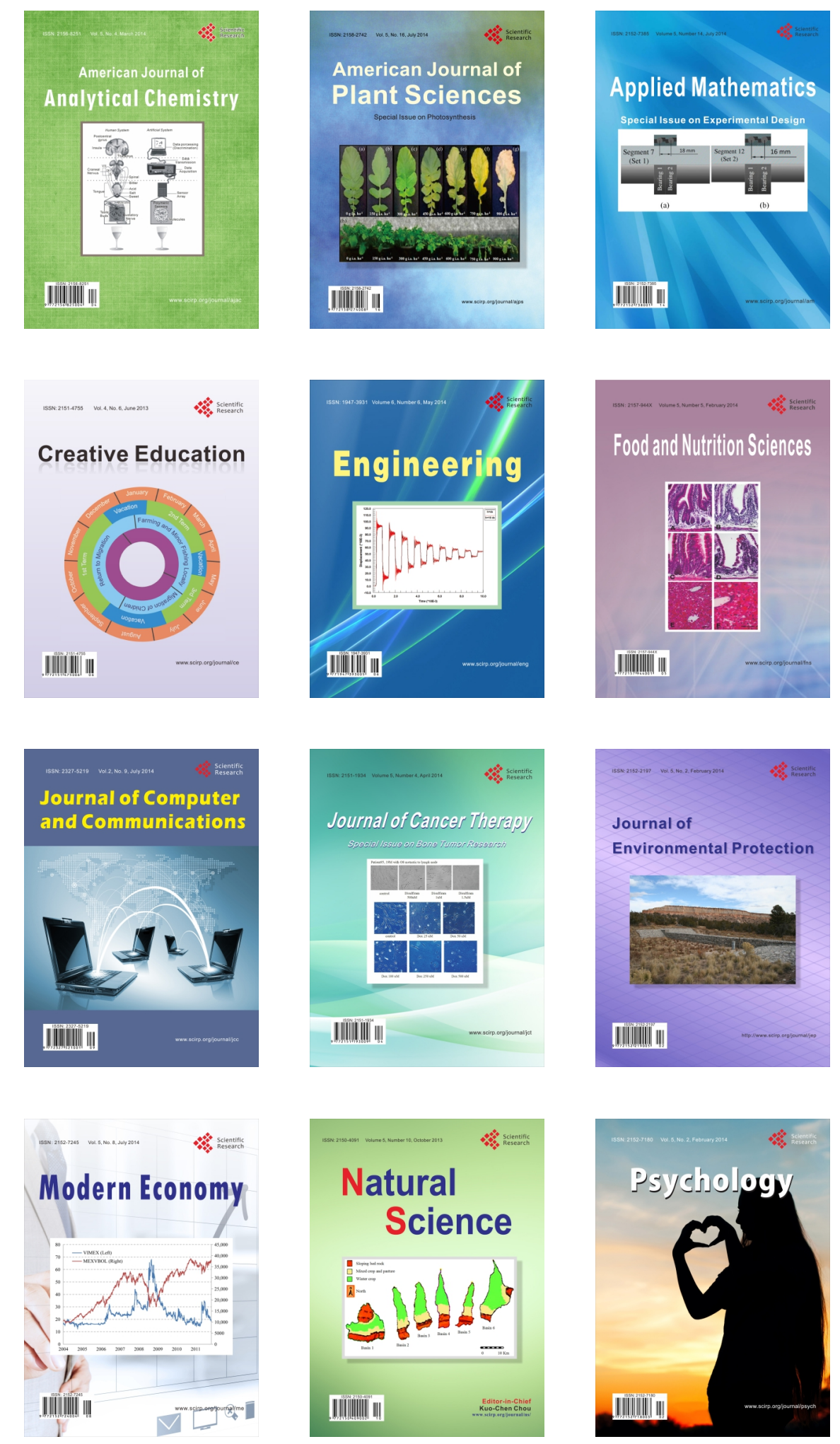